\title{
National politics matter! - Die Suche nach einer europäischen Verfassung und die Stärkung der nationalen Legitimationsgrundlagen der EU
}

\author{
Timm Beichelt und Anja Thomas*
}

Am Beginn des Verfassungsprozesses der Europäischen Union stand die Erklärung von Laeken, die das Ziel formulierte, angesichts der Unzulänglichkeiten des soeben ausgehandelten Nizza-Vertrags die Europäische Union ,demokratischer, transparenter und effizienter" zu gestalten. ${ }^{1}$ Die bald darauf folgende Einberufung des Europäischen Konvents nahm dabei Impulse einer Debatte über das Ziel - die ,finalité - der europäischen Integration auf, die im Grunde seit deren Anbeginn geführt worden war, aber seit der ,Kommission Delors “ (1985-1995) merklich an Fahrt aufgenommen hatte. ${ }^{2}$

Viele Wortführer der Diskussion um die Zukunft der Europäischen Union hatten die Ziele der europäischen Integration - Wohlfahrt, Stabilität und Sicherheit - mit einer Relativierung des Nationalstaatlichen zugunsten von föderalistischen Visionen verbunden. Man denke an die Reden und Positionen etwa von Altiero Spinelli, Jean Monnet oder Joschka Fischer. ${ }^{3}$ Gleichzeitig hatte es auch gewichtige Gegenentwürfe gegeben, besonders von staatlichen Akteuren wie Charles de Gaulle oder Margaret Thatcher. ${ }^{4}$ Die heraufziehende Osterweiterung sowie der zunehmend transnationale Charakter wichtiger politischer Herausforderungen in der Klima-, Migrations- oder Kriminalitätsbekämpfung hatten jedoch den Befürwortern einer stärkeren Integration wichtige Argumente geliefert für eine ,immer engere Union der Völker Europas". 5

Die Erklärung von Laeken, die in der Fortsetzung der vorangegangenen Vertragsrevisionen sowohl supranationale als auch intergouvernementale Lösungswege für institutionelle Reformen vorzeichnete, stilisierte den Europäischen Konvent als „Scheideweg“ und „entscheidenden Moment" der „europäischen Geschichte“.6 Die von den Staats- und Regierungschefs selbst niedergeschriebene Verfassungsrhetorik und die perzipierte Legitimitätskrise der Europäischen Union gab dem Präsidenten des Europäischen Konvents Valéry Giscard d'Estaing und seinem Präsidium schließlich die Möglichkeit, der Regierungs-

\footnotetext{
* Prof. Dr. Timm Beichelt, Professur Europa-Studien, Europa-Universität Viadrina, Frankfurt (Oder). Anja Thomas, Wissenschaftliche Mitarbeiterin, Jean Monnet Lehrstuhl für Politikwissenschaft, Forschungsinstitut für Politische Wissenschaft und Europäische Fragen, Universität zu Köln.

1 Erklärung von Laeken zur Zukunft der Europäischen Union, in: Europäischer Rat: Schlussfolgerungen des Vorsitzes. Europäischer Rat (Laeken) 14. und 15. Dezember 2001, SN 300/1/01, Anlage I, S. 19-26, hier S. 21.

2 Vgl. Craig Parsons: A Certain Idea of Europe, Ithaca/London 2003; Rüdiger Hohls/Iris Schröder/Hannes Siegrist (Hrsg.): Europa und die Europäer. Quellen und Essays zur modernen europäischen Geschichte, Stuttgart 2005 .

3 Vgl. entsprechend Altiero Spinelli: The Growth of the European Movement since the Second World War, in: Michael Hodges (Hrsg.): European Integration, Harmondsworth 1972 [Original 1957], S. 43-68; Frederic J. Fransen: The Supranational Politics of Jean Monnet. Ideas and Origins of the European Community, Westport 2001; Heinrich August Winkler: Integration oder Erosion. Joschka Fischers „Humboldt-Rede“: Absicht und Wirkung, in: Rüdiger Hohls/Iris Schröder/Hannes Siegrist (Hrsg.): Europa und die Europäer. Quellen und Essays zur modernen europäischen Geschichte, Stuttgart 2005, S. 469-474

4 Vgl. Ronald Tiersky (Hrsg.): Euro-skepticism. A Reader, Lanham 2001.

5 So lautet die berühmte Formulierung aus der Präambel des EU-Vertrags in der Fassung des Vertrages von Lissabon, in: Amtsblatt der EU, Nr. C 115 vom 9. Mai 2008, S. 13-45, hier S. 16.

6 Erklärung von Laeken, 2001, S. 19.
} 
konferenz ein Jahr später nicht nur eine unbestimmte Wunschliste, sondern einen in sich geschlossenen Text als Entwurf eines, Verfassungsvertrages ${ }^{67}$ vorzulegen. Verbunden war damit bei vielen beteiligten Akteuren und in Teilen der wissenschaftlichen Öffentlichkeit nicht zuletzt die Hoffnung, einen Verfassungsmoment zu begründen, der der Union alternative Legitimationsquellen erschließen würde, von denen eine die öffentlichkeitswirksame Ratifikation des Textes durch Referenden und die nationalen Parlamente sein würde. ${ }^{8}$

Aus der Vogelperspektive ist es daher nicht verfehlt, im Anfangsmoment des Verfassungsprozesses bei einer Reihe von Akteuren eine Suche nach weiterer Relativierung der nationalstaatlichen Legitimationsgrundlagen europäischer Politik auszumachen, insbesondere durch den Ausbau einer genuin EU-europäischen Repräsentation, die Aufwertung des europäischen Parlamentarismus und die Beteiligung der Bürger durch die Erwägung von Instrumenten der direkten Partizipation.

Im Jahre 2009 erscheint es allerdings keineswegs so, als ob sich diese von einem Teil der europäischen Eliten ursprünglich gehegten Hoffnungen verwirklicht hätten. Mehrere realpolitische Ereignisse weisen vielmehr in die entgegengesetzte Richtung. Zwei äußerst mühsame Regierungskonferenzen, drei gescheiterte Ratifizierungsreferenden in Frankreich, den Niederlanden und Irland sowie eine allgemein konstatierte ,Europamüdigkeit ${ }^{9} 9$ stehen in deutlichem Gegensatz zur Verfassungseuphorie der Konventsphase. Hinzu kommt der Eindruck, der seit Herbst 2008 grassierenden Finanz- und Wirtschaftskrise werde zu einem maßgeblichen Teil auf der nationalen Ebene zu begegnen versucht, wenn man etwa an die Reaktionen vieler Gewerkschaftsbewegungen, an die Einführung von 17 verschiedenen Abwrackprämien in EU-Mitgliedstaaten ${ }^{10}$ sowie an die im Wesentlichen national orientierten Subventionswellen in vielen Staaten denkt.

Viele Interpreten haben diese Entwicklung bedauert und die Verlangsamung des Ratifizierungsprozesses als problematisch für den weiteren Gang der europäischen Integration angesehen. In unserem Beitrag möchten wir dagegen die Position einnehmen, die zögerliche Inkraftsetzung von Verfassungsvertrag und Lissabon-Vertrag entspreche den realpolitischen Grundlagen der gegenwärtigen Integrationsphase. In diesem Sinne könnten der gescheiterte Verfassungsprozess und die schwierigen Verhandlungen zum Lissabon-Vertrag zu einer Klärung der aktuell vorherrschenden legitimatorischen Gewichte in der Entwicklung der Union beitragen und beigetragen haben. Selbst ein endgültiges Scheitern des Lissabon-Vertrags müsste dann nicht als Blockade oder gar Endpunkt der Integrationsentwicklung gelesen werden.

Eine genaue Betrachtung des Lissabon-Vertrags verdeutlicht, dass das neue Institutionengefüge dem vorab formulierten Ziel eines umfassenden ,gemeinsamen Ansatz[es] [...] bei allen grenzüberschreitenden Fragen" in einer recht spezifischen Weise gerecht wird. ${ }^{11}$ Anders als nach der Einheitlichen Europäischen Akte und dem Maastricht-Vertrag zeichnet sich der Integrationsverlauf der jüngeren Zeit vorrangig dadurch aus, dass die Regierungen in vielen Feldern auf die Kontinuität ihrer angestammten Kompetenzbereiche achten. Im

7 Europäischer Konvent: Entwurf eines Vertrags über eine Verfassung für Europa, Konventsdokument CONV 850/03 vom 18.07.2003.

8 Vgl. Ulrike Liebert: Der Verfassungsvertrag: Ein Fortschritt für die demokratische Legitimität in der Europäischen Union, in: Mathias Jopp/Saskia Matl (Hrsg.): Der Vertrag über eine Verfassung für Europa. Analysen zur Konstitutionalisierung der EU, Baden-Baden 2005, S. 383-410, hier S. 405-408.

9 Beim Eintippen des Begriffs ,Europamüdigkeit“ in die Suchmaschine „Google“ ergeben sich immerhin 3.150 direkte Einträge (Zugriff am 07.05.2009).

10 Die Information entstammt einem öffentlichen Vortrag des französischen Botschafters in der Bundesrepublik, Bernard de Montferrand, in Frankfurt (Oder) am 29.04.2009.

11 Siehe Erklärung von Laeken, 2001, S. 20-21. 
Prozess der europäischen Systemwerdung haben sie einen zentralen Ort behauptet, für den sie von vielen ausgewiesenen Befürwortern einer immer weiteren Vertiefung gerade nicht vorgesehen gewesen waren. Damit hat der Verfassungsprozess, der von seinen Unterstützern eine weitere Stärkung supranationaler Elemente nach sich ziehen sollte, in Wirklichkeit die nationalen Fundamente der Europäischen Union noch einmal klarer zu Tage treten lassen.

\section{Zur Rolle der Nationalstaaten im Gefüge der EU}

Welche Rolle nimmt der Nationalstaat in der wissenschaftlichen Debatte um die Verfassungsphase der europäischen Integration ein? Der Beginn des Verfassungsprozesses im Jahre 2001 fiel in eine Zeit, in der sich die theoretische Auseinandersetzung mit der europäischen Integration in einem tiefgreifenden Wandel befand. In den ersten Integrationsjahrzehnten war immer dieselbe Frage hin- und hergewälzt worden: Welche Gründe veranlassen souveräne Staaten und Regierungen, Teile ihrer politischen Verfügungsgewalt - und welche? - an die transnationale Ebene abzugeben? Seit den späten 1980er-Jahren rückte daneben allerdings die Erörterung des Charakters der politischen Prozesse, Politikfelder und Institutionen auf europäischer Ebene. ${ }^{12}$ Der Fokus der theoretisch inspirierten Debatte lag fortan weniger auf dem , supranationalen' oder , intergouvernementalen' Charakter der Integration als auf der Gestalt und Funktionsweise von Netzwerken und Institutionen im „Mehrebenensystem". 13

Selbstredend fußte die Änderung des wissenschaftlichen Fokus nicht allein in der erschöpfenden Behandlung der Frage, ob nationale oder supranationale Akteure beziehungsweise Institutionen als treibende Kräfte des Integrationsprozesses anzusehen seien. Viele Vertreter der einzelnen Schulen standen in regem Austausch untereinander, sodass es bald mehr „Modifikationen“ und „Brückenschläge“ gab als vormals an schmalen Erklärungsansätzen existiert hatten. ${ }^{14}$ Eher als in der wissenschaftlichen Selbstreferenz beruhte die Verlagerung auf einer Reihe von realen Entwicklungen, die einigen Schulen der europäischen Integration gewissermaßen die Grundlagen entzogen. Auch zehn Jahre nach Maastricht war zu erkennen, dass nationale Regierungen nicht nur als treibende Kräfte des Integrationsprozesses, sondern auch des Brüsseler Alltagsgeschäfts zu werten waren. Dies schwächte jene Positionen, die die Auflösung der Nationalstaaten in einer höheren Weltordnung vorhersahen oder für wünschenswert hielten. ${ }^{15}$

Aus der zunehmenden Erklärungsnot einiger der weiterhin auf supranationale Integrationsdynamiken setzenden Theorien folgte allerdings keine geradlinige Rückkehr zu intergouvernementalen Ansätzen. Vielmehr stiegen solche Modelle im Kurs, die den Regierungen einen nicht nur nachrangigen Ort im Entscheidungsgefüge der Europäischen Union zusicherten. So gewann die Vorstellung der Europäischen Union als politischem System an Bo-

12 Thomas Diez/Antje Wiener: Introducing the Mosaic of Integration Theory, in: Antje Wiener/Thomas Diez (Hrsg.): European Integration Theory, Oxford 2004, S. 1-21, hier S. 7.

13 Vgl. z.B. Markus Jachtenfuchs: The Governance Approach to European Integration, in: Journal of Common Market Studies 2/2001, S. 245-264; Arthur Benz: Multilevel Governance - Governance in Mehrebenensystemen, in: Arthur Benz (Hrsg.): Multilevel Governance - Regieren in komplexen Regelsystemen. Eine Einführung, Wiesbaden 2004, S. 125-146.

14 Hans-Jürgen Bieling/Marika Lerch (Hrsg.): Theorien der europäischen Integration, Wiesbaden 2005, S. 143.

15 Otfried Höffe: Demokratie im Zeitalter der Globalisierung, München 1999; Ulrich Beck/Edgar Grande: Das kosmopolitische Europa. Gesellschaft und Politik in der Zweiten Moderne, Frankfurt (Main) 2004. 
den. ${ }^{16}$ Auch in der Governance-Forschung gab es Positionen, die dezidiert eine fortdauernd gewichtige Rolle der nationalen Regierungen herausstrichen. ${ }^{17}$

Der EU-Verfassungsprozess barg dabei irritierende Zusatzschwierigkeiten für die Situationsanalyse. Einerseits signalisierte er auf der rhetorischen Ebene, dass Politik in Europa nach wie vor auf integrationsdynamischen Fundamenten zu ruhen hatte. Andererseits hatte sich die Zahl der real zur Option stehenden Leitbilder im Sinne verwirklichbarer Verfassungs- und Staatsverständnisse deutlich reduziert. ${ }^{18}$ Bereits zu Beginn der Verfassungsdebatte handelte es sich im Grunde um eine Rückkehr altbekannter Rezepte. Joschka Fischers ,Humboldt-Rede ${ }^{19}$ lief auf ein föderales Konzept hinaus; der französische Premierminister Lionel Jospin hatte andere Vorstellungen von einer „fédération d'états-nations“ “. ${ }^{20}$ Um das Spannungsverhältnis zwischen europäischem Föderalismus und einem auf Nationalstaaten basierenden Europa hatte sich bereits die Integrationsdebatte der 1950er- und 1960er-Jahre gedreht.

Die Wiedergeburt der nationalen Regierungen im Mehrebenensystem stand allerdings unter anderen Vorzeichen als in der Epoche der ,großen Männer' mit europageschichtlichem Auftrag. ${ }^{21}$ Die Vergemeinschaftung war zwischenzeitlich wenigstens insoweit fortgeschritten, als für die Legitimation neuer EU-politischer Strukturen nicht mehr allein die Staatsund Regierungschefs ausreichten. Auch Entscheidungen, die Kernfragen der Legitimität europäischer Politik betrafen, hatten sich dem Modus der nicht-hierarchischen Steuerung zu unterwerfen. Regieren, verstanden als Prozess ,,bewusster politischer Zielbestimmung und Eingriffe zur Gestaltung gesellschaftlicher Zustände“, 22 orientierte sich primär an den Präferenzen von Bürgern und gesellschaftlichen Akteuren. Das Repräsentationsmodell politischer Herrschaft hatte ,Gesellschaft bekommen'.23 Die Entscheidung, die Beratungen über die künftige Verfasstheit der Europäischen Union einem ,Konvent` anstelle einer Regierungskonferenz zu überlassen, lässt sich zweifellos in diesen Kontext einordnen.

Im Rückblick muss der ,Verfassungsprozess “ - samt den anschließenden Auseinandersetzungen um den Lissabon-Vertrag - als doppelt codierte Entwicklung verstanden werden. Die Suche nach mehr „Demokratie, Transparenz und Effizienz“, ${ }^{24}$ wie es in der Erklärung von Laeken hieß, bezog sich zunächst auf plausible Ziele im Rahmen des Mehrebenensystems. Daneben stand jedoch die Frage nach der Selbsttransformation eben jenes Regimes, falls sich neu gestärkte Legitimationskanäle etablieren würden. Nicht umsonst standen daher

16 Simon Hix: The Political System of the European Union, New York 1999; Jürgen Hartmann: Das politische System der Europäischen Union. Eine Einführung, Frankfurt (Main) 2001.

17 John Peterson: Policy Networks, in: Antje Wiener/Thomas Diez (Hrsg.): European Integration Theory, Oxford 2004, S. 117-135.

18 Heinrich Schneider: Deutsche Europapolitik: Leitbilder in der Perspektive - Eine vorbereitende Skizze -, in: Heinrich Schneider/Mathias Jopp/Uwe Schmalz (Hrsg.): Eine neue deutsche Europapolitik? Rahmenbedingungen - Problemfelder - Optionen, Bonn 2002, S. 69-131.

19 Joschka Fischer: Vom Staatenbund zur Föderation - Gedanken über die Finalität der Europäischen Union. Vortrag an der Humboldt-Universität zu Berlin am 12. Mai 2000, FCE 12/00 (Spezial 2).

20 Lionel Jospin: „Discours de Lionel Jospin sur l'avenir de l'Europe élargie“ (Paris, 28 mai 2001), abrufbar unter: http://www.ena.lu/discours-lionel-jospin-avenir-europe-elargie-paris-28-mai-2001-010005644.html (letzter Zugriff: 07.05.2009).

21 Beate Kohler-Koch/Thomas Conzelmann/Michèle Knodt: Europäische Integration - Europäisches Regieren, Wiesbaden 2004, S. 29-34.

22 Markus Jachtenfuchs/Beate Kohler-Koch: Governance in der Europäischen Union, in: Arthur Benz (Hrsg.): Governance - Regieren in komplexen Regelsystemen. Eine Einführung, Wiesbaden 2004, S. 77-101, hier S. 78.

23 Gunnar Folke Schuppert: Der Staat bekommt Gesellschaft. Warum die Bilder „Rückzug“ und „Zerfaserung“ nicht weiterhelfen, in: WZB-Mitteilungen 121, September 2008, S. 15-17.

24 Erklärung von Laeken, 2001, S. 22. 
besonders Institutionen der EU-Ebene im Fokus der Konventsdebatten, etwa wenn es um die Ausweitung der Kompetenzen des Europäischen Parlaments oder das reale oder symbolische Machtpotenzial des neu einzurichtenden Amtes des Präsidenten des Europäischen Rates oder des Präsidenten der Kommission ging. ${ }^{25}$

\section{Prinzipien der Legitimation im Vertrag von Lissabon}

Welche Ebenen der Legitimitätsgenerierung lassen sich im Hinblick auf den europäischen Kontext differenzieren? Eine solche Beurteilung ist eng verknüpft mit der Frage nach der Natur der Europäischen Union, entsprechend vielschichtig ist die theoretische Diskussion. Die meisten Ansätze lassen sich jedoch auf einige wenige Vektoren demokratischer Legitimierung reduzieren. ${ }^{26}$ Im Hinblick auf den Vertrag von Lissabon lassen sich entsprechend drei normative Prinzipien benennen: (1) die direkte parlamentarische Repräsentation auf europäischer Ebene, (2) die Repräsentation durch nationale Vertreter, (3) die direkte Partizipation von Bürgern und organisierter Zivilgesellschaft auf EU-Ebene. Jeder dieser Legitimationskanäle birgt in sich eine Input- und Output-Dimension von Legitimität. ${ }^{27}$ Die nun folgende Argumentation zeichnet diese drei Legitimationsdimensionen in ihren Grundzügen nach. Damit verbunden ist die Aussage, dass der Vertrag von Lissabon nicht zwischen den verschiedenen Prinzipien auswählt, sondern letztlich alle Dimensionen zu beeinflussen versucht. Die von uns unterstellte Betonung der Legitimation durch nationale Elemente ist also als Ausdruck einer relativen Gewichtsverschiebung zu werten.

\section{Parlamentarische Repräsentation auf EU-Ebene}

Der Vertrag von Lissabon hält mit Art. 10 EUV-Liss ${ }^{28}$ einen eigenen Artikel bereit, der das Prinzip der repräsentativen Demokratie als grundlegend für die Arbeitsweise der Union bezeichnet. Neben der unmittelbaren Vertretung der Unionsbürger im Europäischen Parlament nennt der Vertrag die indirekte Repräsentation der Bürger einzelner Staaten durch nationale Parlamente, die ihrerseits Regierungen bestimmen und damit ,ihren Bürgerinnen und Bürgern Rechenschaft ablegen müssen“. ${ }^{29}$ Damit stellt der Vertrag einerseits die beiden Ebenen einander gleich und folgt damit der Generallinie von EUV-Liss und AEUV, eine doppelte Legitimationsbasis bei einzelnen Bürgern sowie bei den Mitgliedstaaten zu sehen.

Andererseits wird eher verschleiert, dass die beiden Varianten der ,repräsentativen Demokratie" sich auf den realpolitischen Ebenen hinlänglich unterscheiden. Auf der EU-Ebene sind zwar wesentliche Elemente von Repräsentation gegeben, vor allem die Verantwortung gewählter Repräsentanten vor einem von diesen Repräsentanten abgebildeten Elektorat. ${ }^{30}$

25 Claus Giering: Mutige Einschnitte und verzagte Kompromisse - das institutionelle Reformpaket des EU-Konvents, in: Claus Giering (Hrsg.): Der EU-Reformkonvent - Analyse und Dokumentation, CD-Rom, Gütersloh 2003; Joachim Schild: Die Reform der Kommission: Vorschläge des Konvents, in: integration 4/2003, S. 493500; Philippe de Schoutheete: Die Debatte des Konvents über den Europäischen Rat, in: integration 4/2003, S. 468-482.

26 Markus Jachtenfuchs/Thomas Diez/Sabine Jung: Which Europe? Conflicting Models of a Legitimate European Political Order, in: European Journal of International Relations 4/1998, S. 409-445; Christopher Lord/ Paul Magnette: E Pluribus Unum? Creative Disagreement about Legitimacy in the EU, in: Journal of Common Market Studies 1/2004, S. 183-202.

27 Ebenda, S. 187.

28 Konsolidierte Fassung des Vertrags über die Europäische Union (EUV-Liss) und die Arbeitsweise der Europäischen Union (AEUV) in der Fassung des Vertrags von Lissabon, in: Amtsblatt der EU, Nr. C 115 vom 9. Mai 2008, S. 1-388.

29 Art. 10 Abs. 2 EUV-Liss.

30 Klassisch Warren E. Miller/Donald E. Stokes: Constituency Influence in Congress, in: American Political Science Review 1/1963, S. 45-56. 
Andere Bestandteile des Repräsentationskonzepts finden sich dagegen gar nicht oder in abgeschwächter Form, zum Beispiel der Bezug des repräsentativen Organs zum „Gesamtinteresse des Volkes" oder die horizontale Beschränkung der Repräsentanten in Form einer Gewaltenteilung. ${ }^{31}$ Die durch die Tagung des Europäischen Rates in Laeken, den Konvent und die Regierungskonferenzen angestrebte Stärkung der Repräsentationsfunktion auf EUEbene konnte sich daher nur auf solche Aspekte beziehen, die im Endeffekt mit den oben angesprochenen föderalen Vorstellungen über die Fortführung des Integrationsprozesses in Einklang stehen.

Im Vertrag von Lissabon schlägt dies insbesondere in der Ausweitung der Legislativrechte für das Europäische Parlament zu Buche. Das bisherige Mitentscheidungsverfahren unter gleichberechtigter Beteiligung des Europäischen Parlamentes, nun symbolisch „ordentliches Gesetzgebungsverfahren“ genannt, wird in 35 zusätzlichen Entscheidungsfällen eingeführt und auf zusätzliche Politikbereiche ausgedehnt, wie beispielsweise auf die Asyl- und Einwanderungspolitik und den Kampf gegen internationale Kriminalität und Terrorismus. ${ }^{32}$ Damit wird es zum am häufigsten anzuwendenden Verfahren in 80 von 256 Gesamtfällen. ${ }^{33}$ Zahlreich bleiben jedoch immer noch Verfahren, die keine Beteiligung des Europäischen Parlamentes erfordern - eine Folge der unvollständigen Gewaltenteilung, deren Regelmäßigkeiten sich eher an Typen von Politikfeldern zeigen als an den Institutionen selbst.

Dafür gewinnt im Vertrag von Lissabon allerdings auch das Verfahren der Zustimmung weiter an Bedeutung. Dieses bedarf der Letztentscheidung des Europäischen Parlaments und weist dem Parlament ein von anderen Instanzen nicht zu beschneidendes Machtpotenzial zu. So wird in Zukunft im Vertrag von Lissabon im Rahmen der Flexibilitätsklausel ${ }^{34}$ die Zustimmung des Europäischen Parlaments notwendig sein. ${ }^{35}$ Darüber hinaus findet sich im Vertrag die Aufhebung der bisherigen Aufteilung des EU-Haushalts in obligatorische und nicht-obligatorische Ausgaben. ${ }^{36}$ Das für die Verabschiedung des Jahreshaushaltsplans entworfene besondere Mitentscheidungsverfahren verleiht dem Europäischen Parlament eine gestärkte Stellung. ${ }^{37}$

Ebenfalls im Sinne föderalistischer Leitbilder sieht der Lissabon-Vertrag nun ausdrücklich die Wahl des Kommissionspräsidenten durch das Europäische Parlament vor, ${ }^{38}$ auch wenn diese Wahl weiterhin auf der Basis eines Vorschlags der Staats- und Regierungschefs erfolgt. Diese sind jedoch gehalten, dabei „,das Ergebnis der Wahlen zum Europäischen Parlament ${ }^{\star 39}$ zu berücksichtigen. Diese zumindest partielle, Wahl' könnte auf lange Sicht auch die Legitimität des Kommissionspräsidenten stärken. ${ }^{40}$ Alle diese genannten Aspekte stärken nicht nur die föderale Komponente, sondern sollen gleichzeitig der Politisierung der Eu-

31 Stichwort ,Repräsentation“ in Manfred G. Schmidt: Wörterbuch zur Politik, 2. Aufl., Stuttgart 2004, S. 612613, hier S. 613.

32 Andreas Hofmann/Wolfgang Wessels: Der Vertrag von Lissabon - eine tragfähige und abschließende Antwort auf konstitutionelle Grundfragen?, in: integration 1/2008, S. 3-20, hier S. 12.

33 Ebenda.

34 Art. 352 AEUV.

35 Vgl. z.B. Elmar Brok/Martin Selmayr: Der, Vertrag der Parlamente' als Gefahr für die Demokratie? Zu den offensichtlich unbegründeten Verfassungsklagen gegen den Vertrag von Lissabon, in: integration 3/2008, S. 217-234, hier S. 227.

36 Art. 314 AEUV.

37 Klemens H. Fischer: Der Vertrag von Lissabon. Text und Kommentar zum Europäischen Reformvertrag, Baden-Baden 2008, S. 423-424.

38 Art. 14 Abs. 1 EUV-Liss.

39 Art. 17 Abs. 7 EUV-Liss.

40 Thierry Chopin: Le traité de Lisbonne: quelles voies pour la politisation de l'Union européenne?, in: Thierry Chopin/Michel Foucher (Hrsg.): L'état de l'Union. Rapport Schuman 2008 sur l'Europe, Paris 2008, S. 51-58, hier S. 52-53. 
ropäischen Union dienen, was wiederum die Möglichkeiten des Europäischen Parlaments ausweiten könnte, sich auf transnationale gesellschaftliche Interessen jenseits des nationalen Wahlkreiszuschnitts zu berufen.

Zuletzt gewinnt das Europäische Parlament im Zuge der Vertragsänderungen nunmehr auch das formale Recht, an der Vorbereitung und Initiative von Regierungskonferenzen im Bereich des ordentlichen und des vereinfachten Änderungsverfahrens teilzunehmen. ${ }^{41}$ Gleichzeitig wird das Europäische Parlament am unter Umständen einzuberufenden Konvent an Vertragsänderungen beteiligt. ${ }^{42}$ Die Beschlussfassung selbst obliegt jedoch weiterhin dem Europäischen Rat. Das Privileg der Systemgestaltung - ein zentrales Element föderaler Ideen - bleibt somit trotz allem weiterhin in den Händen der Mitgliedstaaten. ${ }^{43}$

In der Summe findet sich damit im Vertrag von Lissabon eine Reihe von Regelungen zur Stärkung der direkten Repräsentation der Bürger der Union. Die Neuerungen können einerseits als Fortsetzung der Tendenz aller bisherigen Vertragsveränderungen gesehen werden, die dem Europäischen Parlament stets eine Ausdehnung seiner jeweiligen Kompetenzen zusicherten. Andererseits konnten einige Reformschritte, die noch stärker in Richtung eines Föderalisierungskonzepts gewiesen hätten, bezeichnenderweise nicht durchgesetzt werden. Weder die zwischenzeitlich diskutierte ,Staatenkammer' des Europäischen Parlaments noch die Verlagerung des Initiativrechts in den parlamentarischen Raum erblickten das Licht der Welt. Sie hätten das Gewicht der transnationalen Legitimationsebene in einem Maße gestärkt, die den Regierungsvertretern im Konvent ihre eigene Legitimationsbasis empfindlich beschnitten hätte.

Auch bleibt mit Blick auf die realpolitischen Entwicklungen zweifelhaft, ob die im Lissabon-Vertrag angelegten Reformen zu einer grundsätzlichen Änderung der legitimatorischen Funktion des Europäischen Parlamentes für das politische System der Europäischen Union führen können. Das Parlament wurde zwar weiter in seiner Funktion gestärkt, im Gesetzgebungsprozess zusätzliche Lösungsoptionen zu liefern. In Bezug auf eine fortschreitende Politisierung werden die weiterhin hohen erforderlichen Mehrheiten in den einschlägigen Gesetzgebungsprozessen eine weiter reichende Ausbildung einer Links-rechts-Polarisierung im Europäischen Parlament erschweren. Das Europäische Parlament übernimmt somit zwar mehr und mehr Funktionen, die Parlamente in nationalen parlamentarischen Systemen innehaben. Im Bereich der Funktionen der Inklusion, der Interessenaggregation und Interessenartikulation bleibt es jedoch weiter schwach. ${ }^{44}$

\section{Repräsentation durch nationale Regierungen und Parlamente}

Die Repräsentation der Mitgliedstaaten im Europäischen Rat und im Rat, die den zweiten legitimatorischen Pfeiler von Art. 10 EUV-Liss bildet, ist durch die bisherigen Hinweise auf die eingeschränkte Gültigkeit des Repräsentationsbegriffs für EU-politische Aspekte noch nicht hinreichend charakterisiert. Das komplizierte und dadurch zugleich schwache Regime der horizontalen Gewaltenkontrolle sowie die segmentierten Repräsentationseinheiten namens ,Nationalstaaten' stellen zwar charakteristische Randbedingungen für nationale Akteure im EU-Kontext dar. Ihre Legitimationsposition wird allerdings weniger in diesem Zusammenhang als im Kontext langer beziehungsweise überlanger Legitimationsketten problematisiert. 45

41 Art. 48 EUV-Liss.

42 Hofmann/Wessels: Der Vertrag von Lissabon, 2008, S. 13.

43 Ebenda.

44 Wolfgang Wessels: Das politische System der Europäischen Union, Wiesbaden 2008, S. 150.

45 Erik Oddvar Eriksen/John Erik Fossum (Hrsg.): Democracy in the European Union. Integration through Deliberation?, London/New York 2000. 
Dem nationalen Repräsentationstyp unterliegt die Annahme, der Nationalstaat stelle aufgrund der im Vergleich zur EU-Ebene höheren Homogenität im Hinblick auf politische Kultur und Öffentlichkeit - einen fortdauernd adäquaten Raum für demokratische Meinungsbildungsprozesse und demokratische Kontrolle der gewählten Repräsentanten dar. ${ }^{46}$ Dementsprechend ist es konsequent, nationalen Akteuren und Positionen weiterhin eine starke Rolle in den europäischen Willensbildungsprozessen zuzuweisen. Die im Vertrag von Lissabon gefundenen Lösungen gehen in zwei Richtungen, nämlich die Stärkung von Kontrollrechten für nationale Parlamente sowie die Verteidigung intergouvernementaler Domänen.

Erstere finden sich in ausgeprägter Form im Bereich der Kontrolle der Verhältnismäßigkeit und Subsidiarität, die den nationalen Parlamenten künftig zukommt. Im „Protokoll über die Rolle der nationalen Parlamente in der Europäischen Union“ wird ihnen das Recht eingeräumt, innerhalb von acht Wochen nach Vorlage eines Gesetzgebungsaktes eine Stellungnahme abzugeben. ${ }^{47}$ Je nach Quorum der abgegebenen Stellungnahmen ${ }^{48}$ muss der Entwurf durch die Kommission überprüft werden. ${ }^{49}$ Jedes nationale Parlament hat dabei zwei Stimmen, die gegebenenfalls unter den beiden Kammern aufzuteilen sind. ${ }^{50}$ Wichtig kann auch die Möglichkeit der nationalen Parlamente werden, gegen Gesetzgebungsakte Subsidiaritätsklage ${ }^{51}$ vor dem Europäischen Gerichtshof zu erheben. ${ }^{52}$

Einschlägige rechtswissenschaftliche Kommentare zum Vertrag von Lissabon gehen außerdem davon aus, dass die nationalen Parlamente die ihnen in Artikel 12 EUV-Liss zugesprochenen umfassenden Informations- und Beteiligungsrechte als zwingend ansehen werden und mit dem Lissabon-Vertrag Verfahren gegen säumige europäische Institutionen vor dem Europäischen Gerichtshof zu erwarten sind. ${ }^{53}$ Eine weitere Aufwertung der Rolle nationaler Parlamente im Mehrebenenspiel ist das im Lissabon-Vertrag zugestandene Recht für jedes einzelne nationale Parlament, im Rahmen des vereinfachten Vertragsänderungsverfahrens innerhalb von sechs Monaten ein Veto einzulegen. ${ }^{54}$ Die nationalen Parlamente behalten damit ihr Recht, bei Vertragsänderungen mitzubestimmen. Gleichzeitig werden durch die Veto-Struktur Anreize für die nationalen Parlamentarier gestärkt, sich aktiver in die europäischen Entscheidungsprozesse einzubringen.

Auch die Stärkung der Transparenz der Arbeiten im Ministerrat kann entsprechend gewertet werden. Die Arbeiten des Rates sollen laut Lissabon-Vertrag öffentlich stattfinden, wenn dieser über Gesetzgebungsakte abstimmt. ${ }^{55}$ Alle Ratssitzungen werden dementsprechend in zwei Teile geteilt. Die öffentliche Debatte und Abstimmung über Gesetzgebungsakte kann somit von europäischen und nationalen Medien aufgegriffen und verfolgt werden. Dies wird zweifellos dazu führen, dass Regierungen einer stärkeren Kontrolle ihrer nationalen (oder auch der europäischen) Medien unterliegen und die Verantwortlichkeiten im Gesetzgebungsprozess klarer einzelnen Akteuren zugeordnet werden können. Gleichzeitig

46 Dieter Grimm: Europas Verfassung, in: Gunnar Folke Schuppert/Ingolf Pernice/Ulrich Haltern (Hrsg.): Europawissenschaft, Baden-Baden 2005, S. 177-200; Udo Di Fabio: Die politische Gestalt Europas, in: Frankfurter Allgemeine Zeitung, 22.07.2006, S. 8.

47 Art. 3 und 4 des Protokolls über die Rolle der nationalen Parlamente in der Europäischen Union, in: Amtsblatt der EU, Nr. C 115 vom 9. Mai 2008, S. 203-205.

48 Ein Drittel aller Stimmen oder ein Viertel im Bereich der Justizpolitik und des Strafrechts.

49 Art. 7 Abs. 2 und 3 Subsidiaritätsprotokoll, in: Amtsblatt der EU, Nr. C 115 vom 9. Mai 2008, S. 206-209.

50 Brok/Selmayr: Der, Vertrag der Parlamente', 2008, S. 225-226.

51 Art. 8 Subsidiaritätsprotokoll.

52 Brok/Selmayr: Der, Vertrag der Parlamente', 2008; Hofmann/Wessels: Der Vertrag von Lissabon, 2008.

53 Fischer: Der Vertrag von Lissabon. Text und Kommentar, 2008, S. 123.

54 Art. 48 Abs. 7 EUV-Liss.

55 Art. 16 Abs. 8 EUV-Liss. 
kann dies als Anreiz für nationale Parlamentarier gewertet werden, die europäischen Gesetzgebungsprozesse genauer unter die Lupe zu nehmen.

Die Stützung intergouvernementaler Institutionen und Praktiken verläuft dagegen im Vertrag auf subtilere Art. Zum einen hat eine weitere Vertiefung durch Übergänge zum qualifizierten Mehrheitsentscheid nur an wenigen Stellen stattgefunden. Zum anderen verbleiben viele Instrumente der sozial- und wirtschaftspolitischen Krisenpolitik in nationaler Obhut, was eine enge Koordinierung in diesen Bereichen einschränkt. ${ }^{56}$

\section{Legitimation durch direkte Beteiligung von Individuen und Gruppen}

Angesichts der Probleme der Repräsentation auf europäischer Ebene steht die Einrichtung und Stärkung komplementärer Legitimationswege seit Langem auf der Agenda europäischer Reformen. Zum einen richten sich Bemühungen auf autonome Organisationen der europäischen Gesellschaft, insbesondere in Form von Verbänden sowie der organisierten Zivilgesellschaft. ${ }^{57}$ Zum anderen besteht zusätzliches Legitimationspotenzial hinsichtlich der direkten Beteiligung der europäischen Bürgerinnen und Bürger in den Entscheidungsprozessen der Europäischen Union. Beide Argumentationsstränge verknüpfen sich mit der Hoffnung, durch Inklusion von Interessen eine breitere Legitimitätsbasis und somit eine größere legitimatorische Belastbarkeit von EU-Entscheidungen zu schaffen. Eine substanzielle europäische Bürgerschaft ist schon lange als Voraussetzung für das Funktionieren der repräsentativen Demokratieformen auf der europäischen Ebene gesehen worden. ${ }^{58}$

Entsprechend finden sich diverse neue Bestimmungen zur Beteiligung der Bürger an der Arbeit der EU-Organe im Vertrag von Lissabon. So wird zum ersten Mal die „Gleichheit“" der Bürgerinnen und Bürger festgelegt. ${ }^{59}$ Mit dieser Formulierung nähert sich die Europäische Union der Konstruktion eines Staates, in dem Bürger seitens staatlicher Institutionen als politische und rechtliche Subjekte auf Gleichheitsbasis zu behandeln sind. Gleichzeitig verpflichtet sich die Union dazu, Entscheidungen so „bürgernah wie möglich“ zu treffen. ${ }^{60}$ Den Bürgern wird das Recht zugesprochen, ,am demokratischen Leben der Union teilzunehmen“. ${ }^{61}$ Diese Regelungen genießen jedoch eher deklaratorischen Charakter und ändern zunächst wenig an der Funktionsweise des EU-Systems. ${ }^{62}$

Große Erwartungen sind von Bürgerrechtlern und Nichtregierungsorganisationen in die Grundrechtecharta ${ }^{63}$ gelegt worden. Sie ist im Lissabon-Vertrag zwar nicht mehr in den Text integriert, gilt aber als dem Primärrecht gleichrangig. ${ }^{64} \mathrm{Im}$ Vergleich zum Verfassungsvertrag wird ihre Tragweite allerdings eingeschränkt, indem spezifiziert wird, dass die Zuständigkeiten der Union durch die Charta nicht ausgeweitet werden. ${ }^{65}$ Auch erläutert der Text

56 Fritz W. Scharpf: Regieren in Europa, Frankfurt/New York 1999.

57 Justin Greenwood: Interest Representation in the European Union, Houndsmill 2003; Rainer Eising/Beate Kohler-Koch (Hrsg.): Interessenpolitik in Europa, Baden-Baden 2004.

58 Antje Wiener/Vincent Della Sala: Constitution-making and Citizenship Practice - Bridging the Democracy Gap in the EU?, in: Journal of Common Market Studies 4/1997, S. 595-614, hier S. 597; Peter Graf Kielmansegg: Integration und Demokratie, in: Markus Jachtenfuchs/Beate Kohler-Koch (Hrsg.): Europäische Integration, 2. Aufl., Opladen 2003, S. 49-83.

59 Art. 9 EUV-Liss.

60 Art. 10 Abs. 3 EUV-Liss.

61 Ebenda.

62 Fischer: Der Vertrag von Lissabon. Text und Kommentar, 2008, S. 121.

63 Charta der Grundrechte der Europäischen Union, in: Amtsblatt der EU, Nr. C 303 vom 14. Dezember 2007, S. $1-35$.

64 Art. 6 EUV-Liss.

65 Art. 6 Abs. 1 EUV-Liss und 1. Erklärung zur Charta der Grundrechte der Europäischen Union, in: Amtsblatt der EU, Nr. C 115 vom 9. Mai 2008, S. 337. 
nun, dass die Charta nur im Rahmen der ihr angeschlossenen Erklärungen zu interpretieren ist. ${ }^{66}$ Dies grenzt eine dynamische Auslegung der Grundrechtecharta durch die europäischen Institutionen ein. Polen und Großbritannien werden in gesonderten Protokollen weitreichende ,Opt-Outs' zugesichert. Sie sollen verhindern, dass Rechte zur Wirkung kommen, die nicht im jeweiligen nationalen Recht vorgesehen sind. ${ }^{67}$ Aktive Partizipationsrechte sollen die europäischen Bürger auch über die Möglichkeit einer Bürgerinitiative erhalten. ${ }^{68}$ Mindestens eine Million Unionsbürger aus einer „erheblichen Anzahl von Mitgliedstaaten“ können die Kommission auffordern, im Rahmen ihrer Befugnisse Vorschläge zu unterbreiten, die notwendig sind, um Bestimmungen der Verträge umzusetzen.

Art. 11 EUV-Liss enthält auch die Verpflichtung der Union, neben „Bürgerinnen und Bürgern“ den „repräsentativen Verbänden“ die Möglichkeit zu geben, sich öffentlich zum Unionshandeln auszutauschen. Die Organe werden zu einem ,offenen, transparenten und regelmäßigen Dialog“ mit der Zivilgesellschaft verpflichtet. Darüber hinaus soll die Kommission „,umfangreiche“ Anhörungen mit „Betroffenen“ durchführen. ${ }^{69}$ Als Ziel der kombinierten Maßnahmen kann eine breitere Basis für die Akzeptanz unterstellt werden, damit „die etablierten Regeln auf Grund gesellschaftlich akzeptierter Überzeugungen über die rechtmäBige Quelle von Autorität diskursiv gerechtfertigt werden können““70

Insgesamt ist allerdings festzuhalten, dass mit den genannten Maßnahmen eine letztlich nur begrenzte Ausweitung direktlegitimatorischer Kanäle stattgefunden hat. Reale Bottomup-Beteiligungsrechte bleiben entweder schwach oder genießen überwiegend Absichtscharakter. Die zusätzlichen Partizipationsrechte, die sich aus der europäischen Bürgerinitiative ergeben, dürften vor allem durch organisierte Einrichtungen der Zivilgesellschaft genutzt werden. ${ }^{71}$ Schlussendlich bestehen damit durchaus Chancen für die weitere Entwicklung einer gemeinsamen europäischen bürgerschaftlichen Identität auf der Basis gemeinsamer Normen. Es bleibt jedoch dabei, dass die Aktivierung der Zivilgesellschaft im Sinne eines Topdown-Prozesses betrieben wird, wie er spätestens seit dem Kommissions-Weißbuch zur europäischen Governance bereits jetzt zur politischen Praxis der Europäischen Union gezählt werden darf. ${ }^{72}$

\section{Gewichtsverschiebungen zwischen Verfassungs- und Lissabon-Vertrag}

Die Betrachtung der verschiedenen Legitimationsdimensionen des Vertrags von Lissabon hat gezeigt, dass die - größtenteils bereits im Konvent diskutierten - Versuche der Stärkung der Legitimität der Europäischen Union einer Strategie des , catch-all ‘ gefolgt sind. Die Verfasser der Erklärung von Laeken hatten Erwartungen der Unionsbürger nach mehr demokratischer Beteiligung und Transparenz wahrgenommen. Um die entsprechenden Forderungen zu erfüllen, wurde gewissermaßen mit der Schrotflinte auf all jene Aspekte geschossen, hinsichtlich derer vermeintliche Legitimitätsdefizite zu beheben waren.

Wenn wir uns nun noch einmal den Anfang des Verfassungsprozesses vor Augen führen, lässt sich eine Entwicklung ausmachen: Im Lissabon-Vertrag wurden - im Vergleich zum Verfassungsvertrag - Reformschritte in all jenen Dimensionen zurückgenommen, die nicht auf die Legitimation über nationale Regierungen und Parlamente hinausliefen. Die Referen-

66 Art. 6 Abs. 1 EUV-Liss.

67 Fischer: Der Vertrag von Lissabon. Text und Kommentar, 2008, S. 116.

68 Art. 11 Abs. 4 EUV-Liss.

69 Art. 11 Abs. 1-3 EUV-Liss.

70 Liebert: Der Verfassungsvertrag, 2005, S. 385.

71 Hofmann/Wessels: Der Vertrag von Lissabon, 2008, S. 13.

72 Europäische Kommission: Europäisches Regieren. Ein Weissbuch, KOM (2001) 428. 
dumsniederlagen in Frankreich und den Niederlanden zeigten, dass der Konvent sein Ziel der Stärkung einer echten europäischen Öffentlichkeit, der breitenwirksamen Beteiligung der europäischen Zivilgesellschaft und den ,Sprung in Richtung öffentliche Verfassungsentwicklung ${ }^{673}$ nicht erreicht hat. Die Diskussionen in den betroffenen Mitgliedstaaten verliefen weitgehend anhand nationaler Konfliktlinien und mit Codes und Referenzen nationaler politischer Kultur ab. ${ }^{74}$ Die Hoffnungen auf einen weiter reichenden und legitimatorisch belastbaren gesamteuropäischen Gründungsakt wurden nicht bestätigt.

Schon im Verhandlungsmandat für die Regierungskonferenz 2007 fanden sich keine Elemente der konstitutionellen Symbolik des Verfassungsvertrages mehr. Dies betrifft - neben dem Verfassungsbegriff selbst - die Nennung der Europahymne und Europafahne. Hinzu kam die symbolische Ausgliederung der Grundrechtecharta aus dem Vertrag. Das Primat des Unionsrechts wurde nicht in den Vertrag von Lissabon übernommen. Europäische Gesetzesakte tragen nicht mehr die Bezeichnung ,Europäische Gesetze` oder ,Europäische Rahmengesetze', um den Unterschied zur national-parlamentarischen Souveränität zu markieren. EU-Außenpolitik wird nicht mehr von einem ,Außenminister', sondern von einem ,Hohen Vertreter der Union für die Außen- und Sicherheitspolitik' betrieben. ${ }^{75}$ Das gescheiterte irische Referendum führte außerdem zu einer Zusage seitens des Europäischen Rates vom Dezember 2008 an Irland, die Europäische Kommission auch nach dem Jahr 2014 nicht wie vorgesehen zu verkleinern. ${ }^{76}$ Die damit einhergehende Schwächung der Kommission als Institution wurde hingenommen. In eine ähnliche Richtung wies die Reform des Abstimmungsmodus im Rat, die während der Regierungskonferenz zum Verfassungsvertrag auf das Drängen Polens in den Vertragstext eingeführt wurde. ${ }^{77}$

Gestärkt wurden dagegen im Vertrag von Lissabon die Rechte der nationalen Parlamente. Als größte Neuerung in dieser Hinsicht ist zu werten, dass sie im Titel II zu den ,demokratische[n]" Grundsätzen der Union explizit als wichtige Pfeiler der legitimatorischen Grundlagen der Europäischen Union genannt werden. ${ }^{78}$ Waren sie seit dem Vertrag von Amsterdam im Bereich der Protokolle angesiedelt, werden sie nun sogar vor dem Europäischen Parlament behandelt. ${ }^{79}$ Artikel 12 EUV-Liss stattet die nationalen Parlamente erstmals mit Rechten und Pflichten aus und weist ihnen ausdrücklich eine „Rolle im Rahmen der europäischen Integration“ ${ }^{\prime 80} \mathrm{zu}$. Dies dürfte trotz ihrer tatsächlich noch unterschiedlich ausgeprägten institutionellen Fähigkeiten eine Weichenstellung für die Zukunft bedeuten. ${ }^{81}$

Alles in allem wird im Vertrag von Lissabon die Rolle der Mitgliedstaaten als Inhaber der Kompetenz-Kompetenz noch einmal expliziert, ${ }^{82}$ so wie es in Art. 5 EUV-Liss durch das Prinzip der begrenzten Einzelermächtigung sowie die Grundsätze der „Verhältnismäßigkeit“ und „Subsidiarität“" bereits im Verfassungsvertrag angelegt war.

73 Liebert: Der Verfassungsvertrag, 2005, S. 395.

74 Vgl. für den französischen Fall Joachim Schild: Ein Sieg der Angst - das gescheiterte französische Verfassungsreferendum, in: integration 3/2005, S. 187-200, hier S. 191.

75 Fischer: Der Vertrag von Lissabon. Text und Kommentar, 2008, S. 43-47.

76 Europäischer Rat: Tagung des Europäischen Rates vom 11./12. Dezember 2008 in Brüssel. Schlussfolgerungen des Vorsitzes, 17271/1/08, S. 2.

77 Fischer: Der Vertrag von Lissabon. Text und Kommentar, 2008, S. 81.

78 Ebenda, S. 122-123.

79 Brok/Selmayr: Der, Vertrag der Parlamente‘, 2008, S. 225.

80 Fischer: Der Vertrag von Lissabon. Text und Kommentar, 2008, S. 122.

81 Vgl. zum Zweifel an den institutionellen Fähigkeiten der nationalen Parlamente: Sven Hölscheidt: Formale Aufwertung - geringe Schubkraft: die Rolle der nationalen Parlamente gemäß dem Lissabonner Vertrag, in: integration 3/2008, S. 254-265.

82 Vgl. Art. 4 und 5 EUV-Liss. 


\section{Fazit: Stärkung der nationalen Fundamente in der Mehrebenen-Polity}

Am Ende des mutmaßlichen, Verfassungsjahrzehnts 'scheinen sich die legitimatorischen Gewichte zwischen Befürwortern und Skeptikern einer immer weiteren Vertiefung verschoben zu haben. Der gescheiterte Verfassungsprozess hat die politischen Akteure stärker als bisher gezwungen, ihre jeweiligen Vorstellungen mit Perzeptionen und Präferenzen der Bevölkerungen abzugleichen. Die eigentliche Krux für Befürworter weiterer Vertiefungsschritte besteht nun darin, dass sie - anders als die Vertiefungsgegner - jenseits ihrer jeweiligen nationalen Legitimationsbasis erhöhte Rücksicht auf abweichende Stimmungslagen in den übrigen Mitgliedstaaten nehmen müssen. Französischen oder niederländischen Vertiefungsgegnern reicht es aus, auf die vermeintlich ablehnende Haltung der Bevölkerungen in ihrer eigenen ,Constituency ' zu verweisen. Spanische oder deutsche Vertiefungsbefürworter können dagegen die negativen Referenden (Frankreich, Niederlande, Irland) oder unentschlossene politische Eliten (Großbritannien, Tschechien) nicht übergehen, ohne sich in normativ ambivalenten Argumentationsmustern zu verfangen. Denn wenn das Anliegen des Verfassungsprozesses in der Steigerung einer europäischen repräsentativen Basis oder der adäquateren Einbeziehung gesellschaftlicher Akteure bestand, müssen die geäußerten Präferenzen auch ernst genommen werden.

Es lässt sich somit die These formulieren, der Verfassungsprozess habe aus Sicht der Befürworter einer weiteren Vertiefung - vor allem im Sinne der politischen Vertreter neo-föderalistischer Ideen - einen kontraproduktiven Effekt gehabt. Mindestens bis zum Ende des ,permissive consensus' konnten sie zumindest latent von einer Position der moralischen Überlegenheit aus agieren. Schließlich hatte die europäische Einigung Frieden und Wohlstand über einen lange zerstrittenen Kontinent gebracht. Konkurrierende Positionen konnten als gestrige Haltungen abgelehnt werden, zum Beispiel als realitätsferne französische Großmachtgefühle oder als gouvernantenhafte Handtaschenpolitik von Margaret Thatcher. Vor dem Hintergrund der legitimatorischen Debatte in und nach dem Konvent müssen dieselben politischen Akteure sich heute dafür rechtfertigen, über - durch Referenden legitimierte Misstrauensbekundungen gegen das europäische Projekt hinwegzugehen.

Die Reaktion auf die Wiederbelebung der nationalen Legitimierungselemente ist bei manchen Debattenteilnehmern verhalten bis enttäuscht ausgefallen. ${ }^{83}$ Wir möchten jedoch in eine andere Richtung argumentieren. Wird der Vertrag von Lissabon auf seine unterschiedlichen Legitimierungsebenen hin betrachtet, können gegenüber dem Status quo von Nizza Verbesserungen in letztlich allen Legitimitätsdimensionen verzeichnet werden. Dass sich nationale Elemente als besonders belastbar erwiesen haben, spricht für ihre Vitalität auch und gerade im Hinblick auf die Akzeptanz des Integrationsprozesses als Ganzem. Es wäre demzufolge fahrlässig, diese Entwicklung mit Metaphern wie dem Auftauchen von ,Hindernissen“ oder gar einem ,Scheitern' zu versehen. Vielmehr sollte von einem weiteren Schritt in der Polity-Entwicklung der Europäischen Union ausgegangen werden; einer Polity allerdings, die sich weniger in Richtung einer Quasi-Föderation als in Richtung eines Mehrebenensystems bewegt, dessen fundamentale Legitimitätsbasis zunächst im nationalen Raum angesiedelt bleibt.

Der große Vorteil des Lissabon-Vertrags gegenüber seinen Vorgängerdokumenten bleibt, dass sowohl die Entstehung des Textes sowie seine Inkraftsetzung von ungleich intensiveren öffentlichen Debatten als in der Vergangenheit begleitet wurden. Bezieht man sich auf die

83 Vgl. Ulrike Guérot: Europa wie es sinkt und lacht. Fünf Thesen für eine neue EU-Debatte, in: Internationale Politik 3/2009, S. 52-59. 
Dimension der Legitimität, gibt es nur wenige Gründe, den Status quo gegenüber dem Konvolut des Lissabon-Vertrags vorzuziehen. Allerdings haben sich möglicherweise auch die Gelegenheitsstrukturen für weitere Vertiefungsschritte im Integrationsprozess geändert. Selbst wenn der Vertrag von Lissabon trotz aller Verzögerungen im Laufe des Jahres 2010 in Kraft treten sollte, stehen die Weichen jedenfalls kurzfristig nicht in Richtung einer erneuten Vertiefungsrunde, wie es seit Maastricht nach allen Vertragsveränderungen der Fall gewesen ist.

Die Verfassungsdiskussion in ihrem eigentlichen Sinne dürfte allerdings noch lange nicht als abgeschlossen gelten. Schließlich wurden auf viele zentrale Fragen der demokratischen Legitimation weder im nun vorliegenden Vertragstext noch im Prozess der Inkraftsetzung hinlänglich befriedigende Antworten gefunden. Die Lage im Jahr 2009 unterscheidet sich allerdings insofern von der Laekener Konstellation, als sich bei der Suche nach demokratischer Teilhabe in Europa der Fokus auf die nationale Ebene verschoben hat. Eine stärkere Debatte über die Defizite der dort stattfindenden politischen Prozesse und Diskurse in der Europapolitik kann langfristig auch Grundsteine für weitere Vertiefungsschritte legen.

\section{Governance-Forschung heute und morgen}

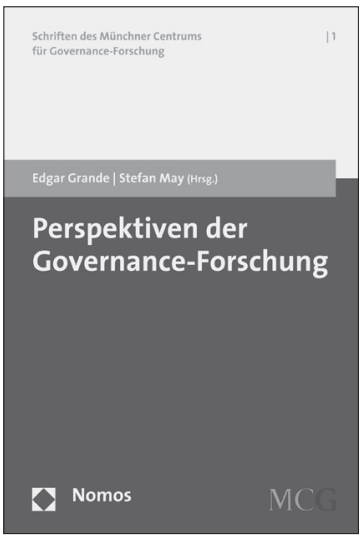

Perspektiven der Governance-Forschung Herausgegeben von Prof. Dr. Edgar Grande und Dr. Stefan May 2009, 91 S., brosch., 14,- $€$, ISBN 978-3-8329-4287-8 (Schriften des Münchner Centrums für GovernanceForschung, Bd. 1)

Ziel des Bandes ist, Stand und Ausblick der Governance-Forschung als auch das Forschungsprogramm des Münchner Centrums für Governance-Forschung interdisziplinär zu diskutieren. Der Band eröffnet zugleich eine Schriftenreihe, in der in den kommenden Jahren die Ergebnisse jener Forschungsarbeit vorgestellt werden sollen. 